\title{
PERCEPÇÃO DOS PROFISSIONAIS DA EQUIPE DE ENFERMAGEM SOBRE O CUIDAR DE PACIENTES EM CUIDADOS PALIATIVOS
}

\section{PERCEPTION OF NURSING STAFF PROFESSIONALS ABOUT THE CARE OF PATIENTS IN PALLIATIVE CARE}

\section{PERCEPCIÓN DE LOS PROFESIONALES DEL EQUIPO DE ENFERMERÍA SOBRE EL CUIDADO DE PACIENTES EN CUIDADOS PALIATIVOS}

Ester Helena de Alcantara ${ }^{1}$, Vanessa Lourenço de Almeida², Márcia Gabriela Nascimento ${ }^{3}$, Maria Betânia Tinti de Andrade ${ }^{4}$, Eliza Maria Rezende Dázio5 ${ }^{5}$ Zélia Marilda Rodrigues Resck ${ }^{6}$.

\section{RESUMO}

Objetivo: compreender a percepção dos profissionais de enfermagem em relação ao cuidar de pacientes em cuidados paliativos. Método: trata-se de um estudo de natureza qualitativa, na abordagem fenomenológica, desenvolvido em uma instituição hospitalar do Sul de Minas Gerais. A técnica de coleta de dados utilizada foi a entrevista aberta, pela questão norteadora: como é para você cuidar de pacientes em cuidados paliativos? A análise de dados foi segundo a compreensão existencialista de Heidegger. Resultados: foram apreendidas três categorias: o ser profissional de enfermagem na assistência a pacientes em cuidados paliativos; ser profissional de enfermagem e o outro: relação interpessoal com o paciente e a família e o ser profissional de enfermagem: a formação e a equipe multiprofissional. Conclusão: o estudo evidenciou situações que levam a expressões de sentimentos e emoções, reconhecimento da humanização e a necessidade de capacitação do profissional de enfermagem atuante na assistência paliativa.

Descritores: Cuidado paliativo; Equipe de enfermagem; Enfermagem.

\section{ABSTRACT}

Objective: to understand the perception of nursing professionals regarding the care of patients in palliative care. Method: it is a qualitative study, in the phenomenological approach, developed in a hospital institution in the South of Minas Gerais. The data collection technique used was the open interview, for the guiding question: how are you going to care for patients in palliative care? The data analysis was according to the existentialist understanding of Heidegger. Results: three categories were seized: the nursing professional in the care of patients in palliative care; being a nursing professional and the other: interpersonal relationship with the patient and the family and the nursing professional: the training and the multi-professional team. Conclusion: the study evidenced situations that lead to expressions of feelings and emotions, recognition of humanization and the need for the training of the nursing professional involved in palliative care.

Descriptors: Palliative care; Nursing team; Nursing.

\section{RESUMEN}

Objetivo: comprender la percepción de los profesionales de enfermería en relación al cuidar de pacientes en cuidados paliativos. Método: se trata de un estudio de naturaleza cualitativa, en el enfoque fenomenológico, desarrollado en una institución hospitalaria del sur de Minas Gerais. Se utilizó la técnica de investigación de entrevista abierta, por la cuestión orientadora: ¿cómo es para usted cuidar de pacientes en cuidados paliativos? El análisis de datos fue según la comprensión existencialista de Heidegger. Resultados: se aprehendieron tres categorías: el ser profesional de enfermería en la asistencia a pacientes en cuidados paliativos; ser profesional de enfermería y el otro: relación interpersonal con el paciente y la familia y el ser profesional de enfermería: la formación y el equipo multiprofesional. Conclusión: el estudio evidenció situaciones que llevan a expresiones de sentimientos y emociones, reconocimiento de la humanización y la necesidad de capacitación del profesional de enfermería actuante en la asistencia paliativa.

Descriptores: Cuidados paliativos; Grupo de enfermería; Enfermería.

${ }^{1}$ Graduada em Enfermagem pela Universidade Federal de Alfenas. ${ }^{2}$ Graduanda em Enfermagem pela Universidade Federal de Alfenas. ${ }^{3}$ Graduada em Enfermagem. Mestranda em Enfermagem pela Universidade Federal de Alfenas. ${ }^{4}$ Graduada em Enfermagem. Mestre em Enfermagem pela Universidade Federal de Minas Gerais. Docente na Universidade Federal de Alfenas. ${ }^{5} \mathrm{Graduada}$ em Enfermagem. Doutora em Enfermagem Fundamental pela Escola de Enfermagem de Ribeirão Preto/USP. Docente na Universidade Federal de Alfenas. ${ }^{6}$ Graduada em Enfermagem. Doutora em Enfermagem pela Escola de Enfermagem de Ribeirão Preto/USP. Docente na Universidade Federal de Alfenas.

Como citar este artigo:

Alcântara EH, Almeida VL, Nascimento MG, et al. Percepção dos Profissionais da Equipe de Enfermagem Sobre o Cuidar de Pacientes em Cuidados Paliativos. 2018;8: e2673. [Access___]; Available in:__. DOI: http://dx.doi.org/10.19175/recom.v8i0.2673 


\section{INTRODUÇÃO}

Cuidados paliativos constituem uma nova modalidade de cuidado fomentada por uma equipe multidisciplinar, que objetiva a melhoria da qualidade de vida do paciente e seus familiares, diante de uma doença que ameace a vida, por meio da prevenção e amenização do sofrimento, identificação precoce, avaliação minuciosa e controle da dor e de sintomas físicos, sociais, psicológicos e espirituais, oferecendo proteção e suporte para viver mais ativo quando possível, até à morte ${ }^{(1-2)}$.

A Organização Mundial da Saúde (OMS) divulgou que, no mundo, o número total estimado de pessoas em cuidados paliativos consiste em cerca de 40 milhões, abrangendo as que estão no estágio inicial, sendo que, aproximadamente $6 \%$ destas são crianças e que, cerca de 20 milhões de pessoas, por ano, necessitam de cuidados paliativos ao final de suas vidas $^{(3)}$.

O aumento em anos da expectativa de vida da população, vem acompanhado de comprometimento das funções orgânicas, concorrendo para o aparecimento de doenças crônicas não transmissíveis e de doenças incapacitantes e, por conseguinte, o aumento da demanda por cuidados paliativos ${ }^{(4)}$.

Mediante a peculiaridade dessa nova modalidade de cuidado, destaca-se a atuação dos profissionais de enfermagem, que oferecem cuidados contínuos, considerando a pessoa nas dimensões biopsicosocioespirituais, não se limitando apenas aos procedimentos técnicos ${ }^{(5)}$.

Corrobora-se ainda, o resgate da essência da Enfermagem, como arte e ciência do cuidar, o que lhes permite compreender que, na assistência a pacientes em cuidados paliativos, mesmo não havendo a cura, é possível oferecer o cuidado para a qualidade de vida, por meio da assistência interdisciplinar e a participação dos familiares ${ }^{(6)}$.

Identifica-se expressivo número de profissionais da equipe de enfermagem com dificuldade de comunicação diante de pacientes em cuidados paliativos, gerando intenso estresse para esses ${ }^{(7)}$. Urgem estudos que resgatem os desafios que as equipes multiprofissionais de cuidados paliativos enfrentam diariamente ${ }^{(7)}$.

Acrescenta-se a isso, a necessidade de inserir, nas Dinâmicas Curriculares de cursos de graduação e de pós-graduação em Enfermagem, disciplinas que abordem a temática dos cuidados paliativos e da morte ${ }^{(8)}$.

O estudo objetivou compreender a percepção dos profissionais da equipe de enfermagem em relação ao cuidar de pacientes em cuidados paliativos.

\section{MÉTODO}

Estudo de natureza qualitativa, abordagem fenomenológica. Realizado em uma instituição hospitalar do Sul de Minas Gerais, que atende, em sua maioria, clientela do Sistema Único de Saúde (SUS), que constitui Centro de Alta Complexidade Oncológica (CACON), possui Unidade de Terapia Renal Substitutiva, Centro de Terapia Intensiva e Unidade de Terapia Intensiva Neonatal, referência para 26 municípios circunvizinhos. Como critério de inclusão, foram considerados enfermeiros e técnicos de enfermagem que atuam na assistência nas unidades de internação clínica médica e cirúrgica, que atendem à pacientes oncológicos e que prestam cuidados paliativos.

Nos setores que prestam assistência em cuidados paliativos, possuem 70 profissionais de equipe de enfermagem, no qual participaram do estudo, 27 desses profissionais. Para garantir o anonimato, os participantes do estudo foram identificados pela letra $\mathrm{P}$ (participantes), seguida de número arábico de um até o último entrevistado, a exemplo: P1, P2...sucessivamente.

Para a coleta dos dados, foi utilizada a entrevista aberta, sendo solicitada, previamente, a autorização para gravação em aparelho eletrônico de áudio e a assinatura do Termo de Consentimento Livre e Esclarecido (TCLE). As entrevistas foram realizadas no mês de dezembro de 2016, individualmente, durante os plantões de cada profissional participante do estudo, em local sem interferências, após a aprovação pelo Comitê de Ética em Pesquisa, Parecer no 1.817.762.

Para análise fenomenológica, optou-se pelo existencialismo de Martin Heidegger, por meio das percepções do enfermeiro entrevistado em suas experiências vivenciadas no dia a dia, compreender seus significados no sentido do ser que cuida de pacientes em cuidados paliativos ${ }^{19-}$ 10).

Assim, a análise fenomenológica foi realizada em três etapas. Iniciou-se com a descrição dos depoimentos dos enfermeiros entrevistados. A segunda tratou-se da redução 
em núcleos de sentido, enfatizando a essência das experiências vivenciadas no dia a dia. E a compreensão, a última etapa da análise, consistiu em interpretar os significados em forma de experiência que expressasse o ser profissional de enfermagem, em relação a si mesmo, ao outro e a profissão(9).

\section{RESULTADOS E DISCUSSÃO}

Dos 27 participantes, 9 eram enfermeiros e 18 técnicos de enfermagem, seis do sexo masculino e 21 do sexo feminino, com idade entre 20 a 60 anos, em sua maioria na faixa etária entre 20 a 40 anos, com filhos e pertencentes à religião católica e evangélica.

Reitera-se que, na análise dos resultados, consideraram-se os princípios heideggerianos, que buscam esclarecer acepções do SER profissional de enfermagem, a partir de sua vivência na assistência a pacientes sob cuidados paliativos. Foram apreendidas, dos depoimentos dos participantes do estudo, três categorias.

\section{Categoria 1: 0 ser profissional de enfermagem na assistência a pacientes em cuidados paliativos}

O presente estudo identificou que os participantes vivenciaram os conflitos diários na assistência aos pacientes em cuidados paliativos, aflorando sentimentos de tristeza, pena, impotência. Tais sentimentos se diferenciaram diante da particularidade de cada profissional, visto que os sentimentos manifestados resultaram de um conjunto de circunstâncias particulares ou influenciadas pelo ambiente.

"É uma situação complicada, triste" (P24).

"Cuidar do paciente em cuidados paliativos, em primeiro momento, pode ser doloroso, sofrido" (P25).

"É uma sensação de pena, de dó, por estar nesta situação" (P4).

"Eu já chorei muito em perder pacientes, meu sentimento como enfermeira é assim: impotente diante da situação e ignorante, mesmo sendo treinada e capacitada" (P7).

Sentimento é a capacidade do ser humano de sentir diante de alguma situação e vivências do cotidiano. Em ambiente hospitalar, sentimentos de tristeza, pena, impotência são constantemente expressos pelos profissionais de enfermagem, advindos do exercício do processo de cuidar de pacientes em cuidados paliativos ${ }^{(11)}$.

$\mathrm{Na}$ formação dos profissionais de saúde, salvar vidas é postulado como a base e a meta para o cuidar, e, quando não mais depende de suas capacidades, apreendeu-se que 0 ser profissional de enfermagem experenciou sentimentos em relação ao outro que os remeteram ao sofrimento. Uma vez que o cuidado paliativo foge do tratamento curativo, tendo como foco o alívio da dor e do sofrimento não apenas físico, mas também psicoespiritual, estendendo-se a atenção à família, o que exige do profissional de enfermagem competências psicoemocionais que, muitas vezes, encontram-se fragilizados.

$\mathrm{Na}$ enfermagem, para o processo de cuidar de pacientes em cuidados paliativos, é fundamental a capacitação do profissional para uma visão integral do paciente, para a valorização do cuidado diante do sofrimento do outro, de maneira a não se limitar às concepções tecnológicas do tratamento ${ }^{(12)}$.

Destarte, há relevância da necessidade das instituições hospitalares oferecerem aporte psicológico aos profissionais de enfermagem e educação continuada sobre esta modalidade de cuidado, para que a equipe disponha de uma estrutura psíquica e emocional equilibrada para intervir juntamente aos pacientes e família ${ }^{(13-}$ 14).

Acrescenta-se também o depoimento que desvela gratificação e prazer por ajudar o outro.

“(...) Mas é bem gratificante, no dia a dia, me sinto grata por cuidar do outro" (P3)

A gratificação em cuidar, demonstrada pela gratidão e o prazer, minimiza o sofrimento no seu campo de trabalho. Reputa-se que o prazer e sofrimento são resultados de causas independentes e circunstanciais do trabalho equilibrante. Corrobora-se que, os profissionais que estão satisfeitos em cuidar do outro procuram por aprendizado e conhecimento, por se instrumentalizar para o atendimento, com vistas à qualidade ${ }^{(15)}$.

Pode-se inferir que a gratidão parte do conceito de reconhecimento de ações prestadas à outra pessoa e remete à forma de agradecimento. Ser grato agrega valores individuais do ser humano, que pode gerar várias linhas de pensamentos e entendimentos. Assim, o ser profissional de enfermagem que vivencia o sentimento de cuidar do próximo sem benefício próprio, sem algo em troca, remete para outros sentimentos como o amor, a fidelidade e a compaixão, que os faz experenciar a gratidão e o prazer em cuidar do outro. 
A importância da dimensão da religiosidade na prática da assistência e no enfrentamento pessoal foi abordada por apenas um dos participantes:

“(...) Só Deus mesmo para dar força para gente" (P16).

Diante das diversidades culturais e costumes religiosos, todo profissional da assistência possui valores diferentes, criam sua própria forma de cuidar e seus próprios princípios de espiritualidade e religiosidade ${ }^{(16)}$.

A espiritualidade não está relacionada a uma doutrina religiosa, a práticas organizadas ou a ensinamentos, enquanto experenciada no campo de trabalho, nem a valores religiosos institucionalizados, mas sim, deve estar associada à compreensão de humanização do trabalho ${ }^{(17)}$.

Para tanto, a espiritualidade para o ser profissional de enfermagem deste estudo, desvelada por P16, é um estado de consciência e entendimento interior que serviu como aporte para o enfrentamento tanto para ele, como por aqueles que se encontravam sob sua assistência. Assim, a espiritualidade fortalece 0 ser profissional a se tornar capaz de ser empático com o paciente, a buscar pela compaixão, essenciais para continuidade do vínculo ao cuidar do paciente em cuidados paliativos.

No entanto, muitas vezes, o profissional de enfermagem se depara com a superficialidade da assistência oferecida, muito aquém da exigência holística requerida para a clientela em cuidados paliativos.

O profissional deste estudo relatou vivenciar o desgaste psicoemocional que o levou à rotinização da assistência manifestada pela frieza no cuidar:

“(...) Você se torna uma pessoa fria, a rotina do dia a dia contribui para um desgaste emocional e psicológico. Vejo a necessidade de um aporte psicológico à equipe de enfermagem, é cansativo, é muito sofrimento" (P5).

A exaustão emocional é estabelecida pela redução ou falta de energia relacionada ao sentimento de esgotamento emocional. A demonstração pode ser física, psíquica ou sua associação concomitante ${ }^{(18)}$.

O ser profissional de enfermagem enfatiza a necessidade de um suporte emocional, uma vez que a intensidade da carga de trabalho e a intensidade do cuidar vivenciadas com pacientes em cuidados paliativos desencadeiam um desarranjo psicológico, que compromete o seu autocontrole e o remete à frieza, cansaço, sofrimento e exaustão emocional.

Assim, fica evidente, no presente estudo, a relação entre o desgaste emocional, com a necessidade contínua de acompanhamento para o profissional, uma vez que o longo tempo de exposição acarreta o seu adoecimento.

\section{Categoria 2: 0 ser profissional de enfermagem e o outro: relação interpessoal com o paciente e a família}

O profissional de enfermagem salienta a comunicação interpessoal como essencial para o cuidar, favorecendo o desenvolvimento de vínculo de confiança com o paciente e a família, conhecer suas dificuldades, medos, anseios, expectativas e seu conhecimento sobre a patologia.

“(...) O paliativo veio para oferecer conforto e qualidade de vida, se viver que viva bem, sempre há o que fazer, conversar, ouvir. Às vezes, na correria do plantão, a gente não conversa e, talvez, seja só isso que ele precisa, quer apenas desabafar" (P7).

A comunicação é essencial no cuidado integral e humanizado porque possibilita identificar e acolher, empaticamente, as necessidades dos pacientes e familiares. 0 profissional, quando utiliza dos meios de comunicação, sejam esses verbais e não verbais, os mesmos favorecem a participação do paciente nas decisões e nos cuidados, possibilitando um tratamento digno ${ }^{(19)}$.

Criar vínculo é fundamental para o cuidar de pacientes em cuidados paliativos, uma vez que, para o sucesso de uma intervenção, é essencial a escuta, o diálogo, a fim de propiciar um ambiente seguro e harmonioso, tanto para o paciente, familiares, como para os profissionais.

Nas entrevistas, os profissionais de enfermagem associaram os cuidados paliativos a procedimentos práticos, dando conotação a um cuidado fragmentado, voltado apenas para a alimentação, higienização e o conforto de posição do indivíduo como apresentado nos trechos seguintes:

“(...) Sempre tem cuidado paliativo para oferecer, não só medicação, mas o conforto, dieta, mudança de decúbito, senão também fica a desejar para ele" (P20).

“(...) pensando nos cuidados, dieta, mudança de decúbito, higienização... é um cuidado especial do paciente" (P19) 
O conforto é o resultado das práticas de enfermagem, seus significados se diferenciam de indivíduo para indivíduo, sendo resultado do equilíbrio do individual e do ambiental. Embora os procedimentos técnicos realizados pela equipe de enfermagem sejam de suma importância na recuperação da saúde dos pacientes, é necessário proporcionar um cuidar integral ao envolver as necessidades humanas básicas, já que a assistência em cuidados paliativos exige a promoção do conforto, abrangendo-o para além de sua dimensão física, e que envolva também os aspectos: psicoespiritual, ambiental e sociocultural ${ }^{(20)}$.

Na relação com o outro, pode-se apreender a empatia nos depoimentos dos profissionais e a importância da humanização, tanto com o paciente como com a família, conforme trecho que se segue:

"Para mim, cuidado paliativo é estar junto ao paciente, oferecer apoio aos familiares, não é somente realizar o seu trabalho técnico e deixar a família de lado. Cuidados paliativos não estão somente na parte de medicamentos, mas também em cuidados direcionados ao paciente e sempre tem que haver um olhar para a família" (P10).

O cuidado humanizado é compreendido como uma oportunidade de trazer novamente as qualidades humanas como a empatia, que tem o sentido de se ter um olhar do mundo, do outro, como sentimentos e opiniões. 0 comprometimento da equipe e da família com o paciente é umas das condições necessárias para a humanização, assim como a relação de equilíbrio entre equipe e família, o que contribui para o envolvimento na terapêutica(21).

Humanizar diz respeito à busca pelo respeito, aceitação do indivíduo na sua multiplicidade, ao envolver seus sentimentos, suas dores, dificuldades, toda a bagagem de conhecimentos e seus próprios valores. E a família colabora para que o tempo de hospitalização seja menor e melhor compreendido ${ }^{(22)}$.

Quando os profissionais de enfermagem resgatam os princípios da humanização no seu processo de trabalho, conseguem buscar sentimentos de sua existência, seus valores, e refletir a sua ação, de modo a se colocar no lugar do outro de maneira holística.

Outro ponto importante que todos referenciaram em seus depoimentos é a associação de cuidados paliativos à terminalidade do paciente, como seguem:

“(...) Porque pensamos, inicialmente, na morte como um estágio final. Porém, no decorrer do tratamento, observo que o mais importante está sendo realizado: é a valorização da vida em sua terminalidade "(P25).

O paciente em fase final de vida é apresentado na literatura, em sua maioria, como paciente terminal, isto é, quando o quadro da doença direciona-se progressivamente ao esgotamento de possibilidades do tratamento curativo, sendo a morte iminente em curto prazo de tempo ${ }^{(23)}$.

A associação de cuidado paliativo à terminalidade, pelos profissionais de enfermagem, pode estar relacionada a dois aspectos que envolvem a dificuldade de reconhecimento de quando iniciar a paliação, o que reflete no conhecimento insuficiente. $E$ o outro aspecto é iniciar a assistência paliativa quando o paciente se encontra em estado tardio da evolução da doença.

$O$ ser profissional de enfermagem reconhece a sua dificuldade ao cuidar de pacientes em cuidados paliativos e à família, destaca a importância do cuidado humanizado e reporta ao enfrentamento da terminalidade, uma vez que o ser humano não está acostumado a lidar com perdas, remetendo à expressão de diferentes sentimentos. Entende que as dificuldades vivenciadas também podem estar relacionadas à falta de conhecimento durante a sua atuação.

\section{Categoria 3: O ser profissional de enfermagem: a formação e a equipe multiprofissional}

Os profissionais de enfermagem demonstraram pouco conhecimento sobre cuidado paliativo e referem-se à necessidade de capacitação, conforme apresentado:

"Eu acho que temos muito ainda a aprender, teríamos que ter treinamento, conhecimento das escalas de dor, manejo com os pacientes em cuidado paliativo e o reconhecimento das fases do luto. Preparar a equipe multidisciplinar é muito importante" (P7).

“(...) porque não foi preparado o paciente, a família e a equipe para poder cuidar do paciente em cuidados paliativos" (P14).

Os depoimentos apontaram para a necessidade de implementação dos Projetos Pedagógicos na inserção de conteúdo sobre cuidados paliativos, nas dinâmicas curriculares, 
ainda abordado com superficialidade, pouco instrumentalizando os futuros profissionais a prestarem assistência aos portadores de doenças crônicas e a entenderem o sentido dos cuidados paliativos ${ }^{(24)}$, visto que, na formação dos profissionais, é necessário contemplar reflexões sobre a finitude e desenvolver as habilidades para a compreensão da existência e do existir, voltando o olhar para o seu semelhante como um ser único, ímpar, com aspectos particulares ${ }^{(25)}$.

A assistência paliativa se torna cada vez mais presente em todos os níveis de atenção de saúde. Ocorre que o conteúdo sobre cuidados paliativos tem sido explorado nas pósgraduações, quando deveria ser abordado nos cursos de graduação, no intuito de preparar o profissional com aptidão no desenvolvimento de seu trabalho.

O ser profissional de enfermagem se revela pouco preparado em sua formação profissional para cuidar de pacientes em cuidados paliativos e de sua família, remetendo para a necessidade de maior entendimento das situações vivenciadas e para o preparo quanto à qualidade da assistência pela equipe multidisciplinar. Torna-se imprescindível a inclusão do tema "cuidados paliativos" nos Projetos Políticos Pedagógicos dos cursos de graduação em enfermagem.

\section{CONSIDERAÇÕES FINAIS}

Os profissionais de enfermagem, na assistência a pacientes em cuidados paliativos, vivenciaram sentimentos de tristeza, pena, impotência que remeteram ao reconhecimento da necessidade de suporte emocional. Em contraponto, vivenciaram a gratificação e o prazer em cuidar do outro, a importância da espiritualidade como suporte para essa modalidade de cuidado.

0 ser profissional de enfermagem reconhece a sua dificuldade ao cuidar de pacientes em cuidados paliativos e assistência à família, face ao enfrentamento da terminalidade. Destacam a importância de se instrumentalizarem para o cuidado humanizado; apontam a empatia e a comunicação verbal e não verbal como instrumentos para o cuidar desses pacientes.

Em relação à formação profissional, revelam-se pouco preparados para o cuidado paliativo, apontando a necessidade de adequações na formação dos profissionais de enfermagem. Dessa maneira, é imprescindível que a equipe envolvida no cuidado esteja treinada e capacitada para que a assistência seja eficiente e qualificada.

A temática "cuidados paliativos" é objeto de muito debate na última década; no entanto, ainda se encontram poucos estudos que apontam para os profissionais envolvidos na assistência, em especial a equipe de enfermagem, que requer o preparo precoce e desenvolvimento de habilidades para este tipo de cuidado.

Assim, sugerem-se novos estudos na temática, resgatando a importância do trabalho em equipe, a interdisciplinaridade e um olhar para os profissionais de enfermagem, os quais se deparam, rotineiramente, com a assistência a pacientes em cuidado paliativo.

Reitera-se também a inclusão do conteúdo "cuidados paliativos" nos Projetos Políticos Pedagógicos dos cursos de graduação em enfermagem.

\section{REFERÊNCIAS}

1. World Health Organization (WHO). National cancer control programmers: policies and managerial guidelines. 2a ed. Geneva: WHO; 2002.

2. Vasques TCS, Lunardi VL, Silveira RS, Gomes GC, Lunardi Filho WD, Pintanel AC. Perception of workers of nursing on the implementation of palliative. Rev Enferm UERJ 2013 [citado em 15 jan 2017]; 21(1):16-21. Disponível em: http://www.epublicacoes.uerj.br/index.php/enfe rmagemueri/article/view/6303/5925

3. World Health Organization. First ever global atlas identifies unmet need for palliative care. Geneva: WHO; 2014.

4. Faria JAM de, Ferreira LG, Vieira MAB, Cosenza NN, Alvarenga PP, Figueiredo PL. Profile of patients with indication of palliative care and admitted at the Júlia Kubitschek Hospital FHEMIG. Rev Méd Minas Gerais 2015 [citado em 15 jan 2017]; 25(1):25-9. Disponível em: http://www.rmmg.org/artigo/detalhes/1732

5. Hermes HR, Lamarca ICA. Cuidados paliativos: uma abordagem a partir das categorias profissionais de saúde. Ciênc Saúde Coletiva 2013;18(9):2577-88. DOI: 10.1590/S141381232013000900012

7. Araújo MMT, Silva MJP. A comunicação com o paciente em cuidados paliativos: valorizando a alegria e o otimismo. Rev Esc Enferm USP 2007; 41(4):668-74. DOI: $10.1590 /$ S0080$\underline{62342007000400018}$

8. Braga FC, Queiroz E. Cuidados paliativos: o desafio das equipes de saúde. Psicol USP 2013; 
24(3):413-29. DOI: $\quad \underline{10.1590 / 50103-}$ 65642013000300004

9. Merighi MAB, Gonçalves R, Rodrigues IG. Vivenciando $O$ período puerperal: uma abordagem compreensiva da Fenomenologia Social. Rev Bras Enferm. 2006;59(6):775-9. DOI: 10.1590/S0034-71672006000600010

10. Fernandes MA. A existência no pensamento de Heidegger. RFMC 2014 [citado em 15 jan 2017]; 2(3):125-142. Disponível em: http://periodicos.unb.br/index.php/fmc/article/vi ew/10350

11. Mota MS, Gomes GC, Coelho MF, Lunardi Filho WS, Sousa LD. Reações e sentimentos de profissionais da enfermagem frente à morte dos pacientes sob seus cuidados. Rev Gaúcha Enferm. 2011;32(1):129-35. DOI: $10.1590 /$ S198314472011000100017

12. Sena ELS, Carvalho PAL, Lauton MAR, Andrade $L M$, Jesus IS. Vivência de uma pessoa com câncer em estágio avançado: um olhar segundo a perspectiva de Merleau-Ponty. Rev Min Enferm. 2013;17(3):635-43. DOI: $\quad \underline{10.5935 / 1415-}$ 2762.20130047

13. Monteiro FF, Oliveira $M$, Vall J. A importância dos cuidados paliativos na enfermagem. Rev Dor. 2010 [citado em 15 jan 2017]; 11(3):242-8. Disponível em: http://files.bvs.br/upload/S/18060013/2010/v11n3/a1470.pdf

14. Viana GKB, Silva HA, Lima AKG, Lima ALA, Mourão CML, Freitas ASF et al. Educational intervention in the nursing team beyond palliative care. J Health Biol Sci. 2018 [citado em 15 jan 2017]; 6(2):165-9. Available in: http://periodicos.unichristus.edu.br/index.php/ih bs/article/view/1458

15. Silveira MH, Ciampone MHT, Gutierrez BAO. Percepção da equipe multiprofissional sobre cuidados paliativos. Rev Bras Geriatr Gerontol. 2014;17(1):7-16. DOI: 10.1590/S1809$\underline{98232014000100002}$

16. Borges DC, Anjos GL, Oliveira LR, Leite JR, Lucchetti G. Saúde, espiritualidade e religiosidade na visão dos estudantes de medicina. Rev Bras Clín Méd. 2013 [citado em 15 jan 2017]; 11(1):611. Disponível em: http://files.bvs.br/upload/ S/1679-1010/2013/v11n1/a3380.pdf

17. Silva Filho ALA, Ferreira MC. O impacto da espiritualidade no trabalho sobre o bem-estar laboral. Psicol Cienc Prof. 2015;35(4):1171-87. DOI: $10.1590 / 1982-3703002482013$

18. Rissardo MP, Gasparino RC. Exaustão emocional em enfermeiros de um hospital público. Esc Anna Nery 2013;17(1):128-32. DOI: 10.1590/S1414-81452013000100018

19. Andrade CG, Costa SFG, Lopes MEL. Cuidados paliativos: a comunicação como estratégia de cuidado para o paciente em fase terminal. Ciênc Saúde Coletiva 2013;19(9):2523-30. DOI: 10.1590/S1413-81232013000900006

20. Lima MPO, Oliveira MCX. Significados do cuidado de enfermagem para familiares de pacientes em tratamento paliativo. Rev Rene. 2015; 16(4):593-602. DOI: $10.15253 / \mathrm{rev} \% 20$ rene.v16i4.2752

21. Reis CCA, Sena ELS, Fernandes MH. Humanização do cuidado nas unidades de terapia intensiva: revisão integrativa. J Res Fundam Care 2016;8(2):4212-22. DOI: 10.9789/2175-5361.2016.v8i2.4212-4222

22. Lopes M, Silva AC, Ferreira AM, Lino AACF. Revisão narrativa sobre a humanização da assistência pela equipe de enfermagem na área oncológica. Gest Saúde 2015; 6(supl 3):2373-90. Disponível em: $10.18673 /$ gs.v0i0. 22457

23. Monteiro MC, Magalhães AS, Féres-Carneiro T, Machado RN. A relação médico-família diante da terminalidade em UTI. Psicol Argum. 2015 [citado em 15 jan 2017]; 33(81):314-29. Disponível

https://www.researchgate.net/publication/ $\underline{318853229}$

24. Oliveira MC, Gelbcke FL, Rosa LM, Vargas MAO, Reis JBG. Cuidado paliativo: visão de enfermeiro de um hospital de ensino. Enferm Foco 2016; 7(1):28-32. DOI: 10.21675/2357707X.2016.v7.n1.661

25. Vicensi MC. Reflection on death and dying in the ICU from a professional perspective in intensive care. Rev Bioét. 2016;24(1):64-72. DOI: $\underline{10.1590 / 1983-80422016241107}$

Nota: O trabalho faz parte de monografia de final de curso.

Recebido em: 15/12/2017

Aprovado em: 16/07/2018

Endereço de correspondência:

Ester Helena de Alcantara

Rua Gabriel Monteiro da Silva, 700, Centro,

CEP:37130-001 - Alfenas-MG - Brasil

E- mail: estheralcantara10@hotmail.com 